\title{
Simulating Complex Calcium-Calcineurin Signaling Network
}

\author{
Jiangjun Cui and Jaap A. Kaandorp \\ Section Computational Science, Faculty of Science, University of Amsterdam, \\ Kruislaan 403, 1098 SJ Amsterdam, The Netherlands \\ \{jcui, jaapk\}@science.uva.nl
}

\begin{abstract}
Understanding of processes in which calcium signaling is involved is of fundamental importance in systems biology and has many applications in medicine. In this paper we have studied the particular case of the complex calcium-calcineurin-MCIP-NFAT signaling network in cardiac myocytes, the understanding of which is critical for treatment of pathologic hypertrophy and heart failure. By including some most recent experimental findings, we constructed a computational model totally based on biochemical principles. The model can correctly predict the mutant $\left(\mathrm{MCIP}^{-/-}\right)$behavior under different stress such as PO (pressure overload) and $\mathrm{CaN}^{*}$ (activated calcineurin) overexpression.
\end{abstract}

\section{Introduction}

In eukaryotic cells, $\mathrm{Ca}^{2+}$ functions as a ubiquitous intracellular messenger regulating a diverse range of cellular processes such as cell proliferation, muscle contraction, programmed cell death, etc $[5,8,12,20]$. Recently it has been recognized that calcium also plays a central role in the control of heart growth through a complex calcium-calcineurin-MCIP-NFAT signaling network (see Fig. 1, please note that the abbreviations used in this paper are listed in the legends of Fig. 1 and in the note of Table 1). The heart responds to physiological and pathological stimuli by hypertrophic growth $[20,21]$. Cardiac hypertrophy is a thickening of the heart muscle (myocardium) resulting in a decrease in size of the ventricles. Prolonged pathological hypertrophy may progress to heart failure and significantly increase the risk for sudden death. Thus deciphering the details of the signaling pathways involved in cardiac hypertrophy and understanding their quantitative dynamics through computational modeling will be critical for devising therapeutic drugs for the treatment of heart disease [21].

As shown in the left-up corner of Fig.1, stress incurs the rise of the concentration of cytosolic $\mathrm{Ca}^{2+}$ (in normal cardiac myocytes, cytosolic $\mathrm{Ca}^{2+}$ concentration rests at a level of less than 200nM and it becomes more than $700 \mathrm{nM}$ under a very strong stress condition), which binds to CaM (4:1). $\mathrm{Ca}^{2+}$-bound CaM binds to $\mathrm{CaN}$ to activate it [13]. $\mathrm{CaN}^{*}$ (i.e., activated $\mathrm{CaN}$ ) can bind to MCIP to form Complex1 $[21,22]$. CaN* can also work as the enzyme to help convert NFAT $^{P}$ into NFAT [11]. Another enzyme GSK3 $\beta$ works in the reverse conversion of NFAT into

M. Bubak et al. (Eds.): ICCS 2008, Part III, LNCS 5103, pp. 110-119, 2008.

(C) Springer-Verlag Berlin Heidelberg 2008 
NFAT $^{P}$, which can bind to 14-3-3 to form Complex3 [2,10]. Such conversion between NFAT and NFAT $^{P}$ with the help of two enzymes (GSK3 $\beta$ and CaN* $^{*}$ ) also happens in the nucleus [7]. NFAT in the cytosol will be imported into the nucleus and $\mathrm{NFAT}^{P}$ in the nucleus will be exported into the cytosol. The nuclear NFAT can initiate the transcription of the hypertrophic genes and the gene encoding MCIP (more precisely, MCIP1, a form of MCIP) $[7,23]$. Both GSK3 $\beta$ and CaN* are shuttled between the nucleus and the cytosol $[7,15]$. As shown in the rightup corner of Fig.1, particular stress such as PO can activate BMK1 [19], which catalyzes the conversion of MCIP into $\mathrm{MCIP}^{P}[1] \cdot \mathrm{MCIP}^{P}$ can be converted into $\mathrm{MCIP}^{P P}$ by GSK3 $\beta$. The reverse conversion of $\mathrm{MCIP}^{P P}$ into $\mathrm{MCIP}^{P}$ is again catalyzed by $\mathrm{CaN}^{*}[22]$. $\mathrm{MCIP}^{P P}$ will bind with 14-3-3 to form Complex2 [1].

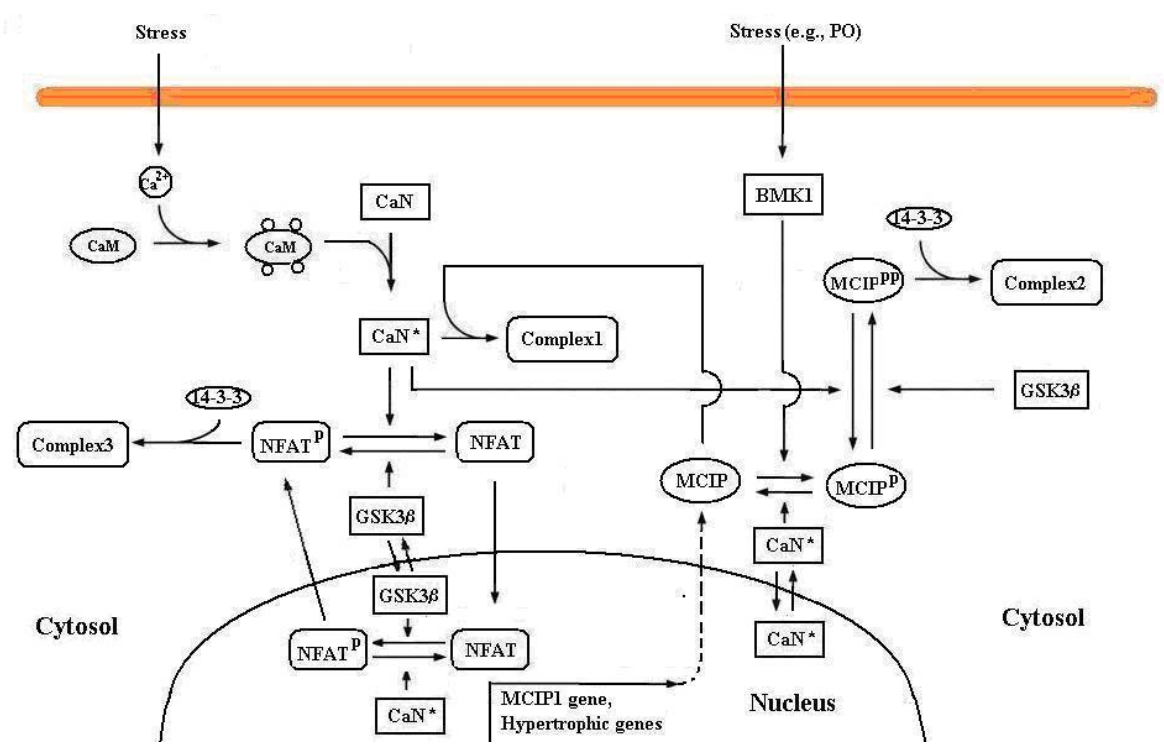

Fig. 1. A schematic graph depicting the $\mathrm{Ca}^{2+}$-calcineurin-MCIP-NFAT signaling networks in cardiac myocytes (for details, please see texts in Introduction). Abbreviations are as follows: calmodulin $(\mathrm{CaM})$; calcineurin $(\mathrm{CaN})$; activated calcineurin $\left(\mathrm{CaN}^{*}\right)$; nuclear factor of activated T-cells (NFAT); phosphrylated NFAT $\left(\mathrm{NFAT}^{P}\right)$; modulatory calcineurin-interacting protein (MCIP); phosphorylated MCIP on serine $112\left(\mathrm{MCIP}^{P}\right)$; phosphorylated MCIP on both serine 112 and serine $108\left(\mathrm{MCIP}^{P P}\right)$; big mitogenactivated protein kinase 1 (BMK1); glycogen synthase $3 \beta$ (GSK3 $\beta$ ); the complex formed by MCIP and calcineurin(Complex1); the complex formed by $\operatorname{MCIP}^{P P}$ and protein 14-3-3 (Complex2); the complex formed by NFAT $^{P}$ and protein 14-3-3 (Complex3); pressure overload (PO); hypertrophic stimuli (stress). The stress of PO is delivered by transverse aortic constriction (TAC).

MCIP1 seems to facilitate or suppress cardiac CaN signaling depending on the nature of the stress (see Fig. 2a). In the case of $\mathrm{CaN}^{*}$ transgenic mice, the knock-out of MCIP1 gene (i.e. MCIP1-/- TG mice) exacerbated the hypertrophic response 
to $\mathrm{CaN}^{*}$ overexpression. Paradoxically, however, cardiac hypertrophy in response to $\mathrm{PO}$ was blunted in normal MCIP1 $1^{-/-}$mice $[9,21]$.

In 2006, Shin et al. [18] published a paper in FEBS Letters using switching feedback mechanism to explain this dual role of MCIP in cardiac hypertrophy. The aim of this paper is to propose a much-extended version of Shin's model by including more recent experimental findings (e.g., $\mathrm{CaN}^{*}$ is imported into the nucleus to function there [7], MCIP ${ }^{P P}$ will associate with protein 14-3-3 [1] and protein 143-3 competes with $\mathrm{CaN}^{*}$ to associate with $\mathrm{NFAT}^{P}$ to form Complex3 [10]). The construction of the model is based on biochemical principles and we use an open source software (Cellerator ${ }^{T M}$ ) to automatically generate the equations. As we will see later, this model can correctly predict the mutant $\left(\mathrm{MCIP}^{-/-}\right)$behavior under different stress such as $\mathrm{PO}$ and $\mathrm{CaN}^{*}$ overexpression.

\section{Computational Model}

\subsection{Cellerator Software}

Cellerator ${ }^{T M}$ is a Mathematica package designed to facilitate biological modeling via automated equation generation [16, 24]. It uses an arrow-based reaction notation to represent biochemical networks and is especially amenable for simulating signal transduction networks.

\subsection{Representation of Relevant Reactions}

The complex $\mathrm{Ca}^{2+}$-calcineurin signaling network shown in Fig.1 can be represented using 17 reactions grouped into four categories (see Table 1) in addition to a transcription control process of MCIP by NFAT.

\subsection{The Equations of the Model}

The ODEs (ordinary differential equations) notation of the set of all relevant reactions consists of 28 equations concerning 28 unknowns including $\mathrm{Ca}(t)$ which denotes the cytosolic $\mathrm{Ca}^{2+}$ concentration. Since calcineurin is unique in its specific responsiveness to sustained, low frequency calcium signals [20], we will assume cytosolic $\mathrm{Ca}^{2+}$ concentration as a constant in the following simulations as Shin et al. [18] did in their simulations. Then we need to further consider modeling the transcription control process of MCIP by NFAT. We have added the following equation to replace the equation of $\mathrm{Ca}(t)$ in our model:

$$
M R N A^{\prime}(t)=k 41 \cdot N F A T n(t)-k 42 \cdot M R N A(t)
$$

where $M R N A(t)$ denotes the mRNA concentration of MCIP and $k 41$ is the control constant, $k 42$ is the degradation constant [28]. Moreover, we need to add an additional production term $(k 43 * M R N A(t))$ and a degradation term $\left(\left(\ln 2 / t_{1 / 2}\right) *\right.$ $M C I P(t))$ in the change rate equation of $M C I P(t)$ where $t_{1 / 2}$ denotes the halflife time constant of MCIP. Thus eventually we have completed building of our 
Table 1. The Representation of Relevant Reactions of The System. Note: Abbreviations and synonyms used in this Table are as follows: $\operatorname{MCIP}^{P}(M C I P p)$; $\operatorname{MCIP}^{P P}(M C I P p p)$ NFAT $^{P}(N F A T p)$; cytosolic NFAT $(N F A T c)$; cytosolic NFAT $^{P}$ $(N F A T p c)$; cytosolic inactive $\mathrm{CaN}(\mathrm{CaNc})$; cytosolic $\mathrm{CaN}^{*}\left(\mathrm{CaNc}^{*}\right)$; cytosolic GSK3 $\beta$ $(G S K 3 \beta c)$; nuclear NFAT $(N F A T n)$; nuclear $\operatorname{NFAT}^{P}$ (NFATpn); nuclear $\mathrm{CaN}^{*}$ $\left(\mathrm{CaNn}^{*}\right)$; nuclear GSK3 $\beta$ (GSK3ßn); protein 14-3-3 (P1433); $\mathrm{Ca}^{2+}$-bound CaM (CaMCa); Complex1 (Comp1); Complex2 (Comp2); Complex3 (Comp3) .

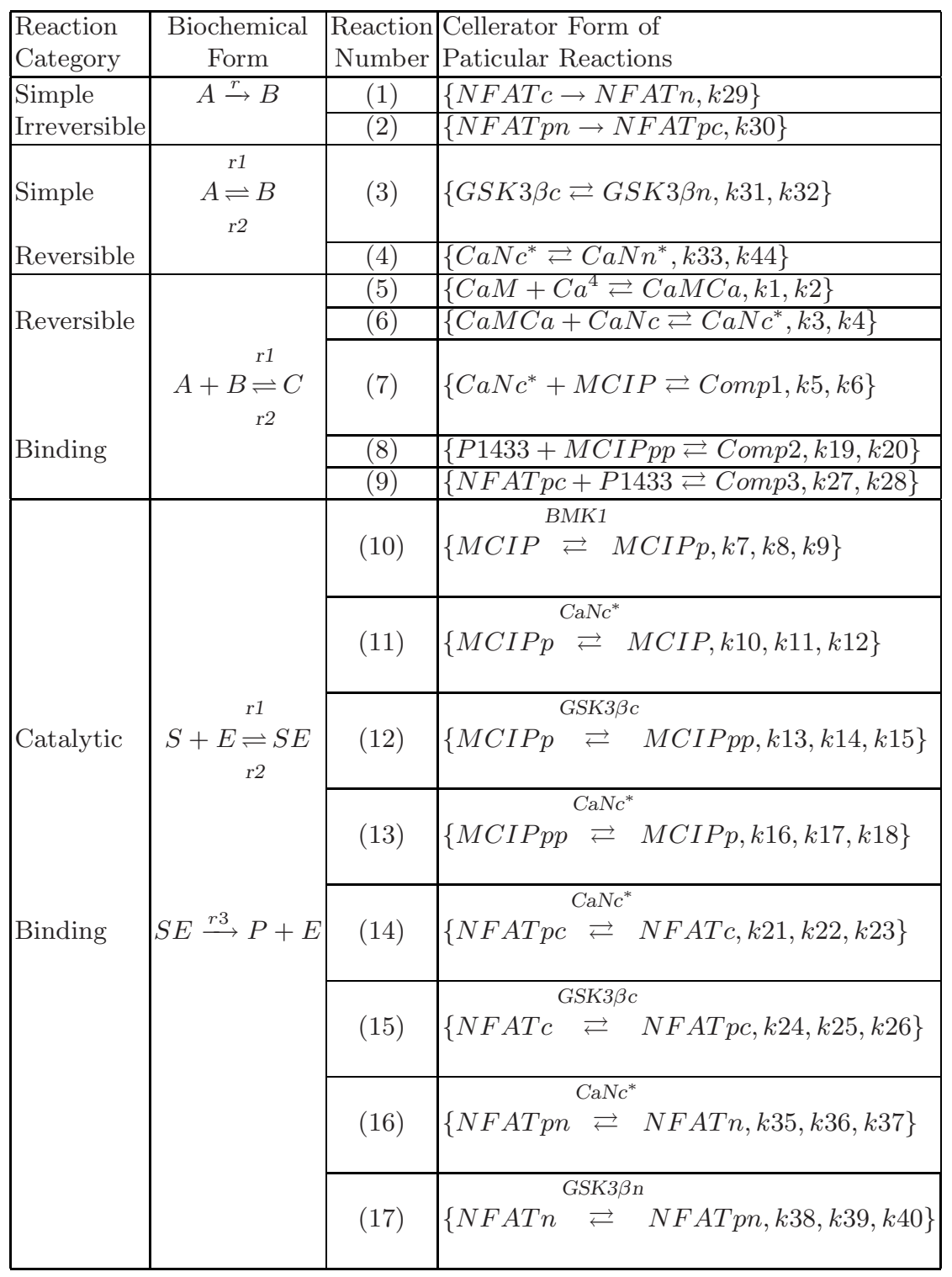


Table 2. Rate Constants and Other Parameters of the Model. Note: $t_{1 / 2}$ denotes the half-life time of MCIP1. $\left[\mathrm{CaN}_{t o t}\right]$ denotes the total concentration of calcineurin. $\left[N F A T_{t o t}\right]$ denotes the total concentration of NFAT.

\begin{tabular}{|c|c|c|c|}
\hline Parameters & Value & Parameter & Value \\
\hline$k 1$ & $0.5 \mu \mathrm{M}^{-4} \cdot \min ^{-1}$ & $k 24$ & $0.1 \mu \mathrm{M}^{-1} \cdot \min ^{-1}$ \\
\hline$k 2$ & $100 \min ^{-1}$ & $k 25$ & $0.15 \min ^{-1}$ \\
\hline$k 3$ & $2760 \mu \mathrm{M}^{-1} \cdot \min ^{-1}[13]$ & $k 26$ & $0.1 \mathrm{~min}^{-1}$ \\
\hline$k 4$ & $0.072 \min ^{-1}[13]$ & $k 27$ & $0.4 \mu \mathrm{M}^{-1} \cdot \min ^{-1}$ \\
\hline$k 5$ & $50 \mu \mathrm{M}^{-1} \cdot \min ^{-1}$ & $k 28$ & $0.1 \min ^{-1}$ \\
\hline$k 6$ & $0.0567 \mathrm{~min}^{-1}[18]$ & $k 29$ & $0.4 \min ^{-1}[15]$ \\
\hline$k 7$ & $5 \mu \mathrm{M}^{-1} \cdot \min ^{-1}$ & $k 30$ & $0.1 \mathrm{~min}^{-1}[15]$ \\
\hline$k 8$ & $0.1 \mathrm{~min}^{-1}$ & $k 31$ & $0.1 \mathrm{~min}^{-1}$ \\
\hline$k 9$ & $0.5 \mathrm{~min}^{-1}$ & $k 32$ & $0.05 \mathrm{~min}^{-1}$ \\
\hline$k 10$ & $0.1 \mu \mathrm{M}^{-1} \cdot \min ^{-1}$ & $k 33$ & $0.114 \min ^{-1}[17]$ \\
\hline$k 11$ & $0.1 \min ^{-1}$ & $k 34$ & $0.0552 \mathrm{~min}^{-1}[17]$ \\
\hline$k 12$ & $0.1 \min ^{-1}$ & $k 35$ & $0.15 \mu \mathrm{M}^{-1} \cdot \min ^{-1}$ \\
\hline$k 13$ & $0.5 \mu \mathrm{M}^{-1} \cdot \min ^{-1}$ & $k 36$ & $0.1 \min ^{-1}$ \\
\hline$k 14$ & $0.5 \mathrm{~min}^{-1}$ & $k 37$ & $0.2 \mathrm{~min}^{-1}$ \\
\hline$k 15$ & $0.1 \mathrm{~min}^{-1}$ & $k 38$ & $0.1 \mu \mathrm{M}^{-1} \cdot \min ^{-1}$ \\
\hline$k 16$ & $0.1 \mu \mathrm{M}^{-1} \cdot \min ^{-1}$ & $k 39$ & $0.1 \min ^{-1}$ \\
\hline$k 17$ & $0.1 \min ^{-1}$ & $k 40$ & $0.1 \mathrm{~min}^{-1}$ \\
\hline$k 18$ & $0.1 \mathrm{~min}^{-1}$ & $k 41$ & $0.02 \min ^{-1}$ \\
\hline$k 19$ & $0.5 \mu \mathrm{M}^{-1} \cdot \min ^{-1}$ & $k 42$ & $0.03 \min ^{-1}$ \\
\hline$k 20$ & $0.1 \mathrm{~min}^{-1}$ & $k 43$ & $0.03 \mathrm{~min}^{-1}$ \\
\hline$k 21$ & $0.15 \mu \mathrm{M}^{-1} \cdot \min ^{-1}$ & $t_{1 / 2}$ & $15 \min [14]$ \\
\hline$k 22$ & $0.15 \min ^{-1}$ & {$\left[\mathrm{CaN}_{t o t}\right]$} & $1 \mu \mathrm{M}[4,18]$ \\
\hline$k 23$ & $0.1 \mathrm{~min}^{-1}$ & {$\left[N F A T_{t o t}\right]$} & $0.017 \mu \mathrm{M}[3,18]$ \\
\hline
\end{tabular}

computational model which consists of 28 ODEs for 28 unknowns. The relevant parameters except parameter $\mathrm{Ca}$, which denotes cytosolic calcium concentration, are listed in Table 2.

\section{Results and Discussion}

Now we have the model, then a natural ensuing question is how to simulate the stress. Similar as Shin et al. did in their paper, we simulate the mild stress (i.e., PO) by setting parameter $C a$ to a smaller constant $(0.2 \mu \mathrm{M})$ (please note that we use a selected steady state as the initial condition for simulating the normally growing heart cells for parameter $C a=0.05 \mu M$. This means that we simulate the increase of the cytosolic $\mathrm{Ca}^{2+}$ concentration incurred by PO by increasing parameter $C a$ from $0.05 \mu \mathrm{M}$ to $0.2 \mu \mathrm{M}$ ). Moreover, at the same time we increase the initial value of $B M K 1(t)$ from $0.012 \mu \mathrm{M}$ to $1.2 \mu \mathrm{M}$ because PO activates BMK1 [19]. The strong stress (i.e., $\mathrm{CaN}^{*}$ overexpression) is simulated by setting parameter $C a$ to a bigger constant $(0.4 \mu \mathrm{M})$ and simultaneously increasing the initial value of $C a N c^{*}(t)$ from $0.0275 \mu \mathrm{M}$ to $0.825 \mu \mathrm{M}$. 
(a)

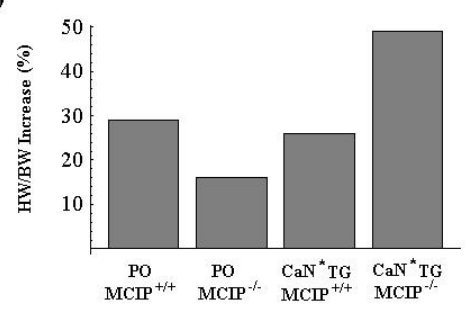

(c)

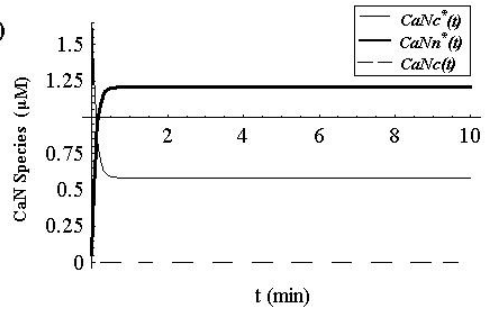

(b)

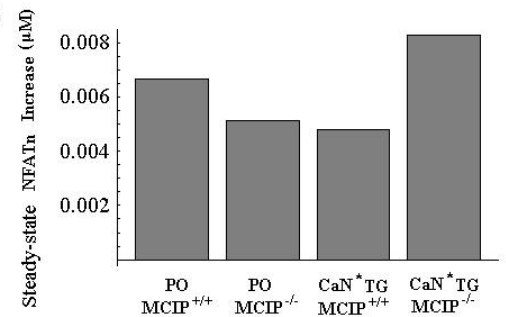

(d)

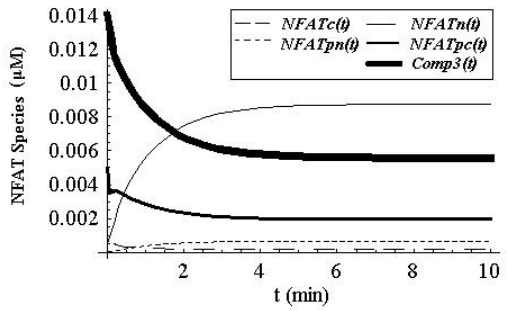

Fig. 2. Hypertrophic response and simulated transient curves for $\mathrm{CaN}^{*} \mathrm{TG}$ animals. (a) The stimuli of pressure overload (PO) caused more severe hypertrophy in normal animals (the first bar) than in $\mathrm{MCIP}^{-/-}$animals (the second bar) whereas the stimuli of $\mathrm{CaN}^{*}$ overexpression (expressed from a muscle-specific transgene) incurred much more $\mathrm{HW} / \mathrm{BW}$ (heart weight normalized to body weight) increase in $\mathrm{MCIP}^{-/-}$transgenic (TG) mice (the fourth bar) than in normal transgenic mice (the third bar). (b) The simulated value increase of steady-state nuclear NFAT under the different stimuli of $\mathrm{PO}$ and $\mathrm{CaN}^{*}$ overexpression. (c) Simulated main CaN species concentration as a function of $t$ in the case of $\mathrm{CaN}^{*}$ overexpression for $\mathrm{MCIP}^{-/-}$animals. Thin solid line: $C a N c^{*}(t)$; thick solid line: $C a N n^{*}(t)$; Dashed line: $C a N c(t)$. (d) Simulated main NFAT species concentration as a function of $t$ in the case of $\mathrm{CaN}^{*}$ overexpression for $\mathrm{MCIP}^{-/-}$ animals. Thin solid line: $N F A T n(t)$; thick solid line: $N F A T p c(t)$; extremely thick solid line: Comp3(t);sparsely dashed line: NFATc $(t)$; densely dashed line: $N F A T p n(t)$.

\subsection{Steady-State Properties}

By numerically solving the equations using the parameters listed in Table 2, simulations show that the system does evolve to a steady state. In Fig. 2b, the steady-state value increase of nuclear NFAT (i.e., $N F A T n^{*}(t)$ ) under the different stimuli for simulated $\mathrm{MCIP}^{+/+}$and $\mathrm{MCIP}^{-/-}$heart cells are shown. By comparison of the first two bars in this Figure, we can see that PO causes greater increase of the steady-state value of nuclear NFAT in simulated normal cells than in simulated MCIP mutant cells. However, the comparison of the third bar with the fourth bar tells us that $\mathrm{CaN}^{*}$ overexpression incurs much less increase of the steady state value of nuclear NFAT in simulated normal cells than in simulated $\mathrm{MCIP}^{-/-}$cells.

\subsection{Transients and Mutant Behavior}

In Fig. 3, critical transient curves in the case of $\mathrm{CaN}^{*}$ overexpression for simulated $\mathrm{MCIP}^{+/+}$cells are shown. From Fig. 3d, we can see that the concentration 


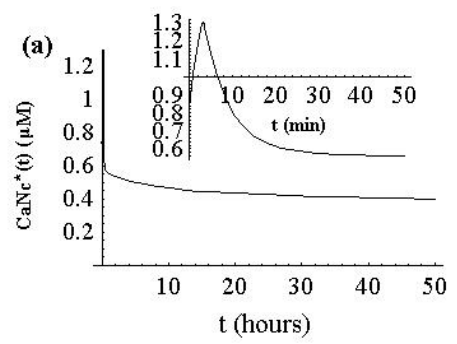

(b)
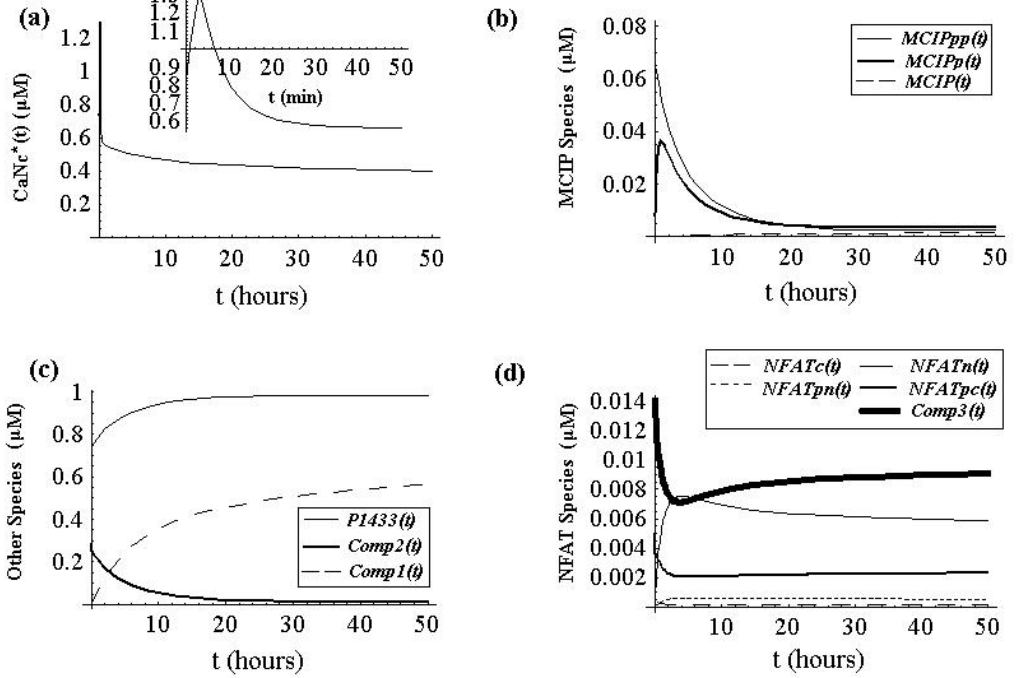

(d)

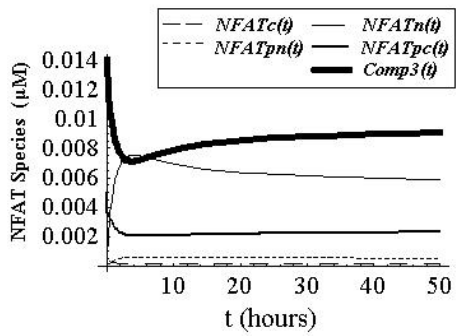

Fig. 3. Simulated transient curves for normal animals under the stimulus of $\mathrm{CaN}^{*}$ overexpression. (a) Simulated $\mathrm{CaNc}^{*}(t)$ (i.e., cytosolic $\mathrm{CaN}^{*}$ ) as a function of $t$. The small figure in the right-up corner shows the detailed change of $\mathrm{CaNc}^{*}(\mathrm{t})$ during the first 50 minutes. (b) Simulated concentration of main MCIP species as a function of $t$. Thin solid line: $M C I P p p(t)$; thick solid line: $M C I P p(t)$; dashed line: $M C I P(t)$; (c) Simulated concentration of some other species as a function of $t$. Thin solid line: P1433(t); thick solid line: Comp2(t); dashed line: $\operatorname{Comp1}(t)$. (d) Simulated main NFAT species concentration as a function of $t$. Thin solid line: $N F A T n(t)$; thick solid line: $N F A T p c(t)$; extremely thick solid line: $\operatorname{Comp} 3(t)$;sparsely dashed line: $N F A T c(t)$; densely dashed line: NFATpn $(t)$.

of nuclear NFAT quickly rises to a peak value of $7.6 \mathrm{nM}$ and then gradually declines (it will eventually rests at $5.3 \mathrm{nM}$ ). Similarly, we can perform numerical simulations for the $\mathrm{MCIP}^{-/-}$animals. Fig. 2c and Fig. 2d show the transient curves of the main CaN species and NFAT species under the stimulus of CaN* overexpression. From Fig. 2d, we can see that nuclear NFAT steadily increases from $0.5 \mathrm{nM}$ to a resting level of $8.8 \mathrm{nM}$.

\subsection{Discussion}

The decrease of Complex2 shown in Fig. 3C means the accelerated dissociation of Complex2 which should produce more $\operatorname{MCIP}^{P P}$ (see Reaction 8 in Table 1). However, from Fig. $3 \mathrm{~b}$, we can see that $\operatorname{MCIP}^{P P}$ is actually decreasing. Since only two reactions (Reaction 8 and 13) can possibly cause the decrease of $\mathrm{MCIP}^{P P}$, the only feasible explanation is that the initial sudden rise of cytosolic CaN* catalyzes the conversion of $\mathrm{MCIP}^{P P}$ to $\mathrm{MCIP}^{P}$ and then to MCIP and the resultant depletion of $\mathrm{MCIP}^{P P}$ promotes the dissociation of Complex2 which also causes the concentration rise of protein $14-3-3$ as seen in Fig. $3 c$. The 
increasing formation of Complex1 shown in Fig. 3c indicates that the MCIP converted from $\mathrm{MCIP}^{P}$ and the newly expressed MCIP associate with cytosolic $\mathrm{CaN}^{*}$. Since in first 50 hours, the total increase of Complex1 concentration (about $0.5 \mu \mathrm{M}$ ) is much greater than the total decrease (less than $0.09 \mu \mathrm{M}$ ) of the concentration of three MCIP species shown in Fig.3b, we can conclude that the greatest source of MCIP consumed in the process of increasing formation of Complex1 is from newly expressed MCIP. The increasing formation of Complex1 also consumes cytosolic $\mathrm{CaN}^{*}$ and realizes the inhibition of MCIP on calcineurin $(\mathrm{CaN})$ activity. In simulated $\mathrm{MCIP}^{-/-}$cells, due to the lack of MCIP, nuclear NFAT increases to a much higher level $(8.8 \mathrm{nM})$ than in $\mathrm{MCIP}^{+/}+$cells $(5.3 \mathrm{nM})$ as shown in Fig.2d. Also it takes much less time for the system to evolve to new steady state.

Similarly, the simulations in the case of PO stress (results not shown here due to the limit of space) show that in normal cells, activated BMK1 promotes the conversion of MCIP to $\mathrm{MCIP}^{P}$ then to $\mathrm{MCIP}^{P P}$ which causes the increase of Comlex2 formation (from $0.28 \mu \mathrm{M}$ to $0.96 \mu \mathrm{M}$ ) and the decrease of free 14-3-3 concentration (from $0.71 \mu \mathrm{M}$ to $0.048 \mu \mathrm{M}$ ). In simulated $\mathrm{MCIP}^{-/-}$cells, due to the existence of large quantity of free 14-3-3, nuclear NFAT increases from 0.5 $\mathrm{nM}$ to a less higher level $(5.6 \mathrm{nM})$ than in $\mathrm{MCIP}^{+/+}$cells $(7.2 \mathrm{nM})$ as shown in Fig.2b.

By comparison of the experimental and simulated hypertrophic response to different stress shown in Fig. 2a and Fig.2b, we can see both two second bars are lower than the corresponding first bars which means that our model does reproduce the seemingly facilitating function of MCIP on hypertrophic response under the stimuli of PO. Moreover, The two fourth bars are higher than the corresponding third bars which means that our model does reproduce the inhibitory function of MCIP on hypertrophic response under the stimuli of CaN* overexpression.

\section{Conclusion}

We have built a computational model for the complex calcium-calcineurinMCIP-NFAT signaling network in cardiac myocytes. Our model can correctly predict the mutant $\left(\mathrm{MCIP}^{-/-}\right)$behavior under different stress such as PO and $\mathrm{CaN}^{*}$ overexpression. Our simulation results suggest that in the case of PO, the seeming facilitating role of MCIP is due to that activated BMK1 promotes the conversion of MCIP to $\mathrm{MCIP}^{P}$ then to $\mathrm{MCIP}^{P P}$ which associates with 14-3-3 to relieve the inhibitory effect of 14-3-3 on hypertrophic response. In the case of $\mathrm{CaN}^{*} \mathrm{TG}$ mice, the overexpressed $\mathrm{CaN}^{*}$ causes the dissociation of Complex2 by promoting the conversion of $\mathrm{MCIP}^{P P}$ to $\mathrm{MCIP}^{P}$ then to MCIP, which associates with $\mathrm{CaN}^{*}$ to inhibit its activity. Moreover, the feedback loop of MCIP expression controlled by NFAT contributes greatly to this inhibition.

Acknowledgements. J. Cui sincerely thanks his group leader Prof. P.M.A. Sloot for sustaining support for his research. We would like to thank Prof. Kyle 
W. Cunningham (Johns Hopkins University, USA) for suggesting the topic. J. Cui was firstly funded by the Dutch Science Foundation on his project 'Mesoscale simulation paradigms in the silicon cell' and later funded by EU on MORPHEX project.

\section{References}

1. Abbasi, S., Lee, J.D., Su, B., Chen, X., Alcon, J.L., Yang, J., Kellems, R.E., Xia, Y.: Protein kinase-mediated regulation of calcineurin through the phosphorylation of modulatory calcineurin-interacting protein 1. J. Biol. Chem. 281, 7717-7726 (2006)

2. Antos, C.L., McKinsey, T.A., Frey, N., Kutschke, W., McAnally, J., Shelton, J.M., Richardson, J.A., Hill, J.A., Olson, E.N.: Activated glycogen synthase- $3 \beta$ suppresses cardiac hypertrophy in vivo. Proc. Natl. Acad. Sci. USA 99, 907-912 (2002)

3. Arron, J.R., Winslow, M.M., Polleri, A., Chang, C.P., Wu, H., Gao, X., Neilson, J.R., Chen, L., Heit, J.J., Kim, S.K., Yamasaki, N., Miyakawa, T., Francke, U., Graef, I.A., Crabtree, G.R.: NFAT dysregulation by increased dosage of DSCR1 and DYRK1A on chromosome 21. Nature 441, 595-600 (2006)

4. Bhalla, U.S., Iyengar, R.: Emergent properties of networks of biological signaling pathways. Science 203, 381-387 (1999)

5. Cui, J., Kaandorp, J.A.: Mathematical modeling of calcium homeostasis in yeast cells. Cell Calcium 39, 337-348 (2006)

6. Dixon, M.: Enzymes, Longman Group Limited London, 3rd edn (1979)

7. Hallhuber, M., Burkard, N., Wu, R., Buch, M.H., Engelhardt, S., Hein, L., Neyses, L., Schuh, K., Ritter, O.: Inhibition of nuclear import of calcineurin prevents myocardial hypertrophy. Circ. Res. 99, 626-635 (2006)

8. Hilioti, Z., Gallagher, D.A., Low-Nam, S.T., Ramaswamy, P., Gajer, P., Kingsbury, T.J., Birchwood, C.J., Levchenko, A., Cunningham, K.W.: GSK-3 kinases enhance calcineurin signaling by phosphorylation of RCNs. Genes \& Dev 18, 35-47 (2004)

9. Hill, J.A., Rothermel, B.A., Yoo, K.D., et al.: Targeted inhibition of calcineurin in pressure-overload cardiac hypertropy: preservation of systolic function. J. Biol. Chem. 277, 10251-10255 (2002)

10. Liao, W., Wang, S., Han, C., Zhang, Y.: 14-3-3 Proteins regulate glycogen synthase $3 \beta$ phosphorylation and inhibit cardiomyocyte hypertrophy. FEBS Journal 272, 1845-1854 (2005)

11. Molkentin, J.D., Lu, J.R., Antos, C.L., Markham, B., Richardson, J., Robbins, J., Grant, S.R., Olson, E.N.: A calcineurin-dependent transcriptional pathway for cardiac hypertrophy. Cell 93, 215-228 (1998)

12. Putney, J.W.: Calcium signaling, 2nd edn. CRC Press, Boca Raton (2005)

13. Quintana, A.R., Wang, D., Forbes, J.E., Waxham, M.N.: Kinetics of calmodulin binding to calcineurin. Biochem. Biophys. Res. Commun. 334, 674-680 (2005)

14. Rothermel, B.A., Vega, R.B., Williams, R.S.: The role of modulatory calcineurininteracting proteins in calcineurin signaling. Trends Cardiovasc. Med. 13, 15-21 (2003)

15. Salazar, C., Hfer, T.: Allosteric regulation of the transcription factor NFAT1 by multiple phosphorylation sites: a mathematical analysis. J. Mol. Biol. 327, 31-45 (2003)

16. Shapiro, B.E., Levchenko, A., Meyerowitz, E.M., Wold, B.J., Mjolsness, E.D.: Cellerator: extending a computer algebra system to include biochemical arrows for signal transduction simulations. Bioinformatics 19, 677-678 (2003) 
17. Shibasaki, F., Price, E.R., Milan, D., McKeon, F.: Role of kinases and the phosphatase calcineurin in the nuclear shuttling of transcription factor NF-AT4. Nature 382, 370-373 (1996)

18. Shin, S.-Y., Choo, S.-M., Kim, D., Baek, S.J., Wolkenhauer, O., Cho, K.-H.: Switching feedback mechanisms realize the dual role of MCIP in the regulation of calcineurin activity. FEBS Letters 580, 5965-5973 (2006)

19. Takeishi, Y., Huang, Q., Abe, J.-i., Glassman, M., Che, W., Lee, J.-D., Kawakatsu, H., Lawrence, E.G., Hoit, B.D., Berk, B.C., Walsh, R.A.: Src and multiple MAP kinase activation in cardiac hypertrophy and congestive heart failure under chronic pressure-overload: Comparison with acute mechanical stretch. Journal of Molecular and Cellular Cardiology 33, 1637-1648 (2001)

20. Vega, R.B., Bassel-Duby, R., Olson, E.N.: Control of Cardiac growth and function by calcineurin signaling. J. Biol. Chem. 278, 36981-36984 (2003)

21. Vega, R.B., Rothermel, B.A., Weinheimer, C.J., Kovacs, A., Naseem, R.H., BasselDuby, R., Williams, R.S., Olson, E.N.: Dual roles of modulatory calcineurininteracting protein 1 in cardiac hypertrophy. Proc. Natl. Acad. Sci. USA 100, 669-674 (2003)

22. Vega, R.B., Yang, J., Rothermel, B.A., Bassel-Duby, R., Williams, R.S.: Multiple domains of MCIP1 contribute to inhibition of calcineurin activity. J. Biol. Chem. 277, 30401-30407 (2002)

23. Yang, J., Rothermel, B., Vega, R.B., Frey, N., McKinsey, T.A., Olson, E.N., BasselDuby, R., Williams, R.S.: Independent signals control expression of the calcineurin inhibitory proteins MCIP1 and MCIP2 in striated muscles. Circ. Res. 87, e61-e68 (2000)

24. http://www.cellerator.info/ 